\title{
Superwinds and the nature of starburst galaxies
}

\author{
Matthew D. Lehnert ${ }^{1}$ \\ Sterrewacht Leiden, Rijksuniversiteit Leiden, \\ Postbus 9513, NL-2300 RA Leiden, Nederland
}

\begin{abstract}
I discuss the observational evidence that starburst galaxies are able to drive galactic-scale outflows ('superwinds') and then argue generally that superwinds must have had an important role in galaxy evolution. To explore the role of feedback from massive stars, I review results suggesting that starbursts seem to obey a limiting IR surface brightness of about $10^{11} \mathrm{~L}_{\odot} \mathrm{kpc}^{-2}$, corresponding to a maximum star-formation rate of about $45 \mathrm{M}_{\odot} \mathrm{yr}^{-1} \mathrm{kpc}^{-2}$ for a 'normal' initial mass function. I conclude by discussing the role of winds in determining this upper-limit and discuss recent results implying that winds might actually escape the galactic potentials in which they reside.
\end{abstract}

\section{Introduction}

The processes that occur during the formation, evolution, and death of massive stars are the engines which drive galaxy evolution. While this statement is obvious enough, this simple fact is often lost in the quest to study the most spectacular objects or to discover the most distant galaxy. Because massive stars play such a fundamental role in galaxy evolution implies that we must have an accurate and detailed understanding of formation and evolution of massive stars and how the processes of their interaction with their surroundings changes as they evolve.

Massive stars are prodigious producers of ionizing photons and mechanical energy and as such they regulate the ionization, physical, and kinematic structure of ISM. The generation of ionizing photons, mechanical energy, and the cosmic rays by massive stars regulate the ISM pressure, and by doing so, perhaps provide the mechanism by which subsequent star-formation is ultimately regulated ('feedback'). Specifically, it is this feedback from massive stars that ultimately balances star-formation against gravitational instability, tidal sheer, and dissipation that give galaxies the characteristics that they are observed to have. In all these areas, the details of stellar evolution play a critical role in understanding how stars regulate all facets of galaxy structure and evolution. Hence determining parameters like mass loss rates, time spent in various evolutionary stages, which stars go supernova, all feed back into our understanding of the structure of the ISM and hence how galaxies evolve.

\footnotetext{
${ }^{1}$ Present address: Max-Plank-Institut für extraterrestrische Physik, Postfach 1603, D-85740 Garching, Germany
} 
Of course, perhaps the best way of directly observing the role of massive stars in driving galaxy evolution is through the study of the most intense starforming galaxies in the universe - starbursts. The purpose of this proceeding is to review the effects of high rates of star-formation on the host galaxy's interstellar medium. Such a discussion demonstrates the central role that massive stars and the effects of stellar evolution have on the properties of galaxies. Of course, demonstrating evolutionary effects directly is difficult. We will take an indirect course. We first show that the preponderance of observational evidence is in favor of starburst galaxies driving galactic scale supernova (and stellar wind)-driven superwinds, that the strength of the wind is dependent on the star-formation rate and distribution, and then discuss the possible implications for self-regulation of star-formation, how superwinds might drive galaxy evolution, and review recent evidence that in fact, superwinds may escape the galaxy potentials in which they reside.

\section{The basic physics of winds}

Superwinds are thought to arise when the star-formation is intense enough to create a region of rare gas of high temperature. This hot gas has an extremely high pressure $\left(T \simeq 10^{7-8} \mathrm{~K}, n_{\mathrm{e}} \simeq 0.01-0.001\right)$, much higher than the ambient ISM pressure and thus is able to push the ambient ISM out preferentially in the direction of the steepest pressure gradient (i.e., the minor axis in disk galaxies). Given sufficient time and mechanical energy input, such a high pressure region will eventually 'break out' due to the various instabilities (mainly dynamical ones) that cause the bubble walls to begin to break-up. When the bubble walls break apart, the wind begins to flow outwards eventually reaching of-order its internal sound speed as a free flowing wind (the effects of gravity can be safely ignored; see Suchkov et al. 1994; Tenerio-Tagle \& Muñoz-Tuñón 1997, 1998). It may also shock and accelerate ambient ISM clouds to velocities of several hundred $\mathrm{km} \mathrm{s}^{-1}$ (Suchkov et al. 1994).

The conditions for establishing such a situation are not well established theoretically and are only hinted at observationally. Obviously a necessary (but not necessarily a sufficient condition) is that the star-formation must be spatially concentrated. This allows the mechanical energy of the stellar winds and supernova remnants to be effectively and efficiently thermalized. If this mechanical energy is not thermalized efficiently, or if lots of cool material is entrained in the wind, it will radiate most of its energy away over a fairly short time scale and thus will not sustain a flow for anything like a sound crossing time over a galactic size scale (which is the minimum condition necessary for driving a galactic scale wind). Thus it is apparent that there are many factors that can keep a galactic scale wind from developing.

\section{Observational properties of winds}

Superwinds are a multi-wavelength phenomenon and the amount of observational evidence at almost all wavelengths has been growing tremendously over the past decade. Different wavelengths probe possibly different phases and phenomenology of the out-flowing wind but all show ample evidence that starburst 


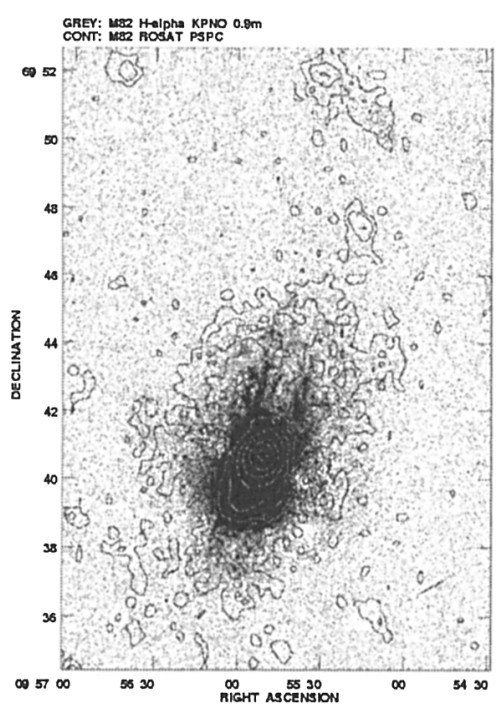

Figure 1. The greyscale is the $\mathrm{H} \alpha$ image and the contours are the ROSATPSPC image of M 82. The spatial coincidence of the $\mathrm{H} \alpha$ and X-ray emission is quite good. The faint ridge of emission to the north is about $11.6 \mathrm{kpc}$ (in projection) above the disk of M 82 (see Devine \& Bally 1999).

galaxies drive superwinds (e.g., Heckman, Armus \& Miley 1990; Lehnert \& Heckman 1996a). This evidence includes: galactic scale bi-polar spatially extended soft X-ray emission along the minor axis of starburst disk galaxies (e.g., M 82, Fig. 1; Watson, Stanger, \& Griffiths 1984; Fabbiano 1988; Bregman, Schulman \& Tomisaka 1995; Moran \& Lehnert 1997; Ptak et al. 1997; NGC 253, Fabbiano 1988; Persic et al. 1998; NGC 1569, Heckman et al. 1995; Della Ceca et al. 1996; NGC 2146, Armus et al. 1995; Della Ceca et al. 1999; NGC 1808, Dahlem, Hartner \& Junkes 1994; NGC 4449, Della Ceca, Griffiths \& Heckman 1997; NGC 3628, Dahlem et al. 1996; Arp 220, Heckman et al. 1996; and for a small sample of edge-on galaxies, Dahlem, Heckman \& Weaver 1998), extended galactic scale optical line emission with evidence for shock-heating (Fig. 2; Lehnert \& Heckman 1996a; Heckman, Armus \& Miley 1990), emission line kinematics that often show split lines, velocity offsets relative to systemic velocity, and broad lines in the most extended emission line gas (Fig. 3; Lehnert \& Heckman 1996a; Heckman, Armus \& Miley 1990), good correlation between ionization state and line width in the extended gas (Lehnert \& Heckman 1996a), nuclear optical emission line gas and X-ray emitting plasma with extremely high pressures (several orders of magnitude higher than ambient ISM pressure in the Milky Way) and with pressure profiles that are consistent with that expected for out-flowing winds (Lehnert \& Heckman 1996a; Heckman, Armus \& Miley 1990; Fabbiano 1988; e.g., all of the X-ray references given previously), and extended polarized radio emission (Dahlem et al. 1996). 


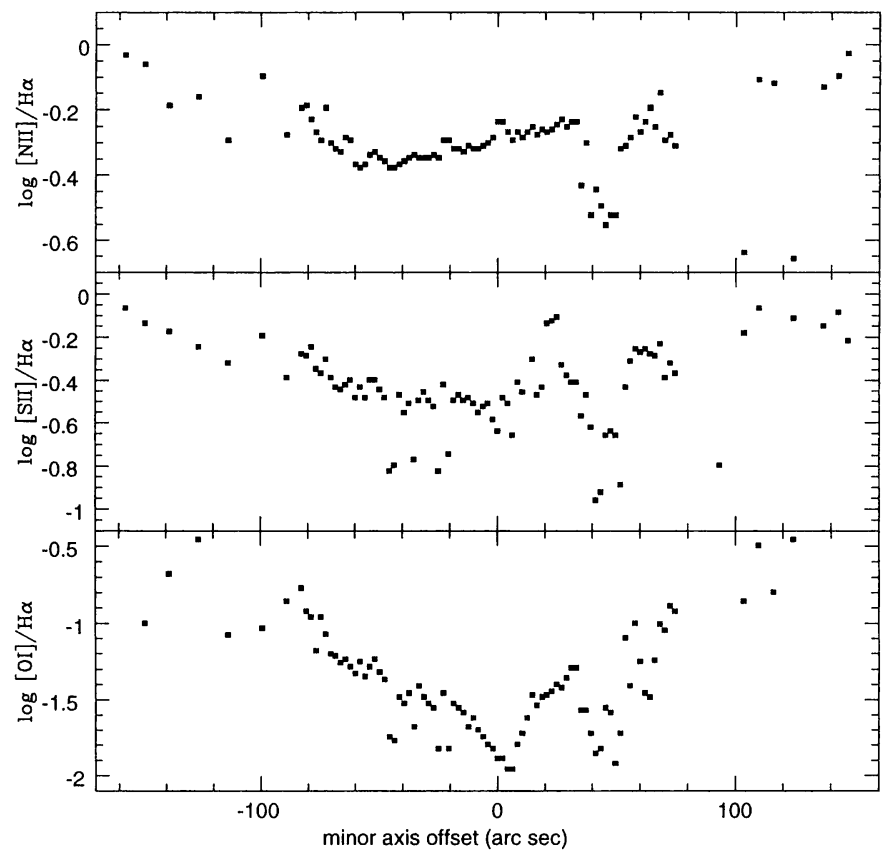

Figure 2. In this three-paneled figure we show the line ratios as a function of height above the disk of M 82. As can be seen, the line-ionization line ratios increase with projected height above the disk consistent with a greater contribution of shock-heating in the gas far above the disk.

Perhaps the best and most direct probes of the wind material are X-rays. As one might recall from the previous section, the wind fluid should be hot in order to provide the high pressure necessary to drive the outflow. However, the predicted X-ray emissivity of the out-flowing material is low (Suchkov et al. 1994, 1996) and yet the observed X-ray luminosities of starburst galaxies are high $\left(10^{38} \mathrm{erg} \mathrm{s}^{-1}\right.$ for a dwarf galaxy like NGC 1569, e.g., Heckman et al. 1995; Della Ceca et al. 1996; to $10^{42} \mathrm{erg} \mathrm{s}^{-1}$ for the almost ultra-luminous IRAS galaxy NGC 3256; Moran, Lehnert \& Helfand 1999). A number of alternatives have been proposed to enhance the X-ray luminosity produced by the wind. The wind could be centrally 'mass loaded' whereby quantities of ambient ISM could be mixed into the wind in or near the starburst (Suchkov et al. 1996; Heckman et al. 1997). Galactic halo clouds, whether pre-existing as might be there as tidal debris of an interaction or tidal encounter or that have been carried out from the disk with the wind material itself, could be evaporated or ripped apart as they are overtaken by or interact with the wind fluid (e.g., Suchkov et al. 1994). Or the wind fluid could drive a shock into a denser volume-filling galactic halo, with the observed X-ray emission arising from the shocked-halo material rather than the wind-fluid itself. Mass loading the wind deep in the starburst nucleus would likely produce a more uniform X-ray and optical line 


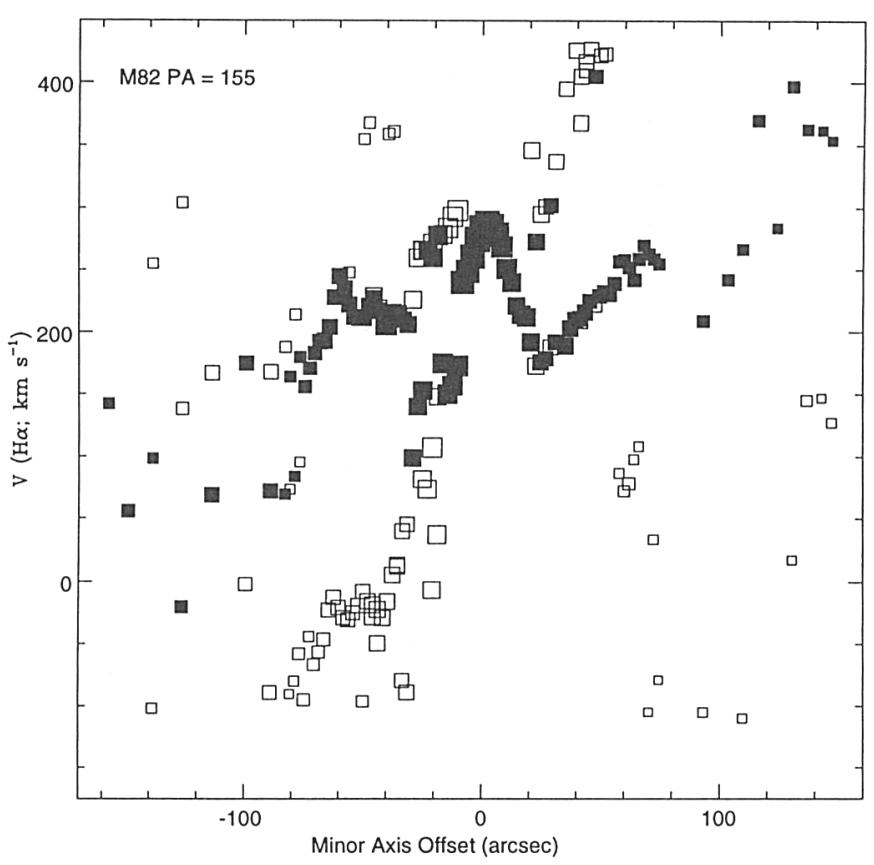

Figure 3. The kinematics of the extended $\mathrm{H} \alpha$ emission along the minor axis of M 82 determined from a long slit spectrum from the KPNO $4 \mathrm{~m}$ telescope. The black squares denote the highest surface gas.

emission in the halos of starbursts (dominated by adiabatic expansion and fluid instabilities), while interactions with halo material would be expected to produce very clumpy emission with large ranges of surface brightness and temperatures. Differentiating between the source of the halo material is less straight-forward, perhaps by using $\mathrm{HI}$ as a tracer of neutral material in the halo or perhaps determining the metal abundance of the high surface brightness regions of $\mathrm{X}$ ray emission.

\section{Conditions necessary for developing outflows}

The theory of superwinds suggests that to generate a wind not only requires active star-formation but in fact that the star-formation must be intense. Intense in this context means that the volume density of energy input must be high enough so that the mechanical energy deposited by stars into the ISM is effectively thermalized before it has a chance to radiate away a significant fraction of its mechanical energy. Lehnert \& Heckman (1996) and Heckman, Armus \& Miley (1990) in studies of galaxies selected from the IRAS survey (i.e., infrared bright) found that it is not only the star-formation rate but other factors like 
the 'warmth' of the IR emission and the ratio of IR to optical luminosity also influenced the observational strength of the wind. Both of these additional factors are related to how enshrouded the starburst region is and the UV heating rate of the dust (which are proportional in some sense to the volume density of the energy input). These results substantiate one of the basic tenets of the superwinds hypothesis.

Specifically it was found that to drive an outflow, a galaxy should have large IR luminosities $\left(L_{\mathrm{IR}}>10^{44} \mathrm{erg} \mathrm{s}^{-1}\right)$, large IR excesses $\left(L_{\mathrm{IR}} / L_{\mathrm{opt}}>2\right)$, and warm far-IR colors $\left(S_{60 \mu m} / S_{100 \mu m} \geq 0.5\right)$. Taking these limits, the IR luminosity function, outflow rates $\propto L_{\mathrm{IR}}$ and using constants of proportionality determined in well-studied examples like M 82 and NGC 253, the local space density of galaxies, and a value of the Hubble time, we derive that superwinds have carried out: $M_{\text {eject }} \approx 5 \times 10^{8} \mathrm{M}_{\odot}$ in metals, and $E_{\mathrm{KE}+\text { thermal }} \approx 10^{59}$ erg per average (Schecter $L^{\star}$ ) galaxy over the history of the universe. Interestingly, these are approximately the mass of metals and the binding energy of an average $\left(L^{\star}\right)$ galaxy. And this estimate is conservative in that it assumes no evolution in the star-formation rate with epoch. Reasonable assumptions about the increasing interaction rate and starburst number density with epoch would only increase our estimates and demonstrates the potentially substantial role outflows have played in galaxy formation and evolution.

\section{Questions surrounding and implications of superwinds}

There are two central questions that studying superwind engenders. Is there some critical star-formation intensity at which the mechanical energy output from the massive stars halts further star-formation? Does the wind ultimately escape the potential of the host galaxy?

Interestingly, it appears that the answer to the first question may be yes. For example, in a study of IR selected starbursts, Lehnert \& Heckman (1996b) found that these galaxies have a 'large-scale' IR surface brightness that appears to have a limit of $\sim 10^{11} \mathrm{~L}_{\odot} \mathrm{kpc}^{-2}$. In a similar study but now including starbursts with a range of selection methods and a wide range of redshifts, Meurer et al. (1997) found a similar limit of $L_{\text {bol }} \approx 2 \times 10^{11} \mathrm{~L}_{\odot} \mathrm{kpc}^{-2}$ (see Weedman et al. 1998, who suggest that this limit may increase with increasing redshift - about a factor of 4 at redshifts $\sim 2-3$ ). This corresponds to a maximum star-formation rate of about $45 \mathrm{M}_{\odot} \mathrm{yr}^{-1} \mathrm{kpc}^{-2}$ for a 'normal' initial mass function. This result should not be over-interpreted. It is not to mean that in all cases, star-formation is stopped at this limit. The cores of some $\mathrm{H}$ II regions and the super-star clusters violate this 'limit'. This is only to suggest that perhaps the feedback from massive stars might provide a global integrated limit to the star-formation rate. On smaller scales, different physical processes undoubtedly dominate compared to those on larger scales which can lead to very high star-formation rates per unit area or volume over limited scales.

In an analysis of this problem, Lehnert \& Heckman (1996b) argued that this limit could plausibly be due to the out-flowing wind providing enough pressure to overcome the hydrostatic pressure and thus halt further star-formation. To show this is plausible, they used M 82 as a test case and showed that star-formation in $\mathrm{M} 82$, which is at or near this limit in the star-formation rate per unit area, 
does provide enough pressure in the wind to balance the hydrostatic pressure of the disk of M 82. However, such an hypothesis awaits further testing (see also the analysis of Meurer et al. 1997).

With the rapid decline in the surface brightnesses of spatially extended $\mathrm{X}$-ray, optical, and radio emission, answering the question of whether or not the wind fluid ultimately escapes the galactic potential is currently a difficult question to answer definitively. Certainly, the model prediction is that it should, but whether or not it actually does depends on where and how the wind is mass-loaded and how much cool material ultimately mixes with the wind fluid. However, some hints at the answer are starting to emerge. For example, Norman et al. (1996) in a study of absorption lines in QSOs projected near the line of sight to NGC 520 and NGC 253 found that it is plausible that the absorption lines they detected along the line of sight to NGC 520 could be associated with an outflow thus suggesting that the wind could reach large distances from the galaxy. However, as they pointed out, this is not a unique interpretation of their results.

Another possible way of addressing this question has recently presented itself. Devine \& Bally (1999) have recently discovered a ridge of correlated $\mathrm{X}$-ray and $\mathrm{H} \alpha$ line emission $11.6 \mathrm{kpc}$ (in projection) above the disk of M 82 (Fig. 1). In an analysis of this ridge of emission, Lehnert, Heckman \& Weaver (1999) have argued that this ridge represents the interaction of the superwind with a dense cloud in the halo of M82 that may be part of the tidal debris from the interaction between M 82/NGC 3077/M 81 (Yun et al. 1993, 1994). The analysis reveals that in order to explain the X-ray properties of the ridge of $\mathrm{X}$-ray $/ \mathrm{H} \alpha$ emission, the out-flowing wind must be hitting the cloud at about $800 \mathrm{~km} \mathrm{~s}^{-1}$ - well in excess of the escape speed at that distance above the plane for M 82. There is little doubt that the wind in M 82 is escaping its galactic potential.

\section{Conclusions}

We have demonstrated that the preponderance of evidence is strongly in favor of starburst driving galactic scale superwinds. In spite of the fact that the existence of superwind is on a firm observational basis, there are still many unanswered questions. We have suggested that winds are likely to have a huge impact during galaxy formation and subsequent evolution but we do not understand the details of that statement. We know that the feedback from massive stars is likely to be important, but how important? What is the nature of the X-ray emission seen in starburst galaxies? The wind in M 82 seems to be able to escape the galactic potential, but is the escape of the wind plasma a general feature of galaxies with superwinds? Was it easier or more difficult in the past for galaxies to drive winds and for these winds to escape the galactic potential? How will the details of stellar evolution and the mechanical energy input change our views of winds and their influence on galaxy evolution? Some of these questions can only be answered definitively when we have a complete understanding of the physics and evolution massive stars including the role of Wolf-Rayet stars in exciting and disturbing the ISM. 
Acknowledgments. I would like to express my sincerest thanks to Karel van der Hucht for his immense patience and understanding in waiting for my contribution.

\section{References}

Armus, L., Heckman, T.M., Weaver, K.A., Lehnert, M.D. 1995, ApJ 445, 666

Bregman, J.N., Schulman, E., Tomisaka, K. 1995, ApJ 439, 155

Dahlem, M., Hartner, G.D., Junkes, N. 1994, ApJ 432, 598

Dahlem, M., Heckman, T.M., Fabbiano, G., Lehnert, M.D., Gilmore, D. 1996, ApJ 461, 724

Dahlem, M., Petr, M., Lehnert, M.D., Heckman, T.M., Ehle, M. 1997, A\&A 320, 731

Dahlem, M., Weaver, K.A., Heckman, T.M. 1998, ApJS 118, 401

Della Ceca, R., Griffiths, R.E., Heckman, T.M. 1997, ApJ 485, 581

Della Ceca, R., Griffiths, R.E., Heckman, T.M., MacKenty, J.W. 1996, ApJ 469, 662

Della Ceca, R., Griffiths, R.E., Heckman, T.M., Lehnert, M.D., Weaver, K.A. 1999, ApJ 514, 772

Devine, D., Bally, J. 1999, ApJ 510, 197

Fabbiano, G. 1988, ApJ 330, 672

Heckman, T.M., Armus, L., Miley, G.K. 1990, ApJS 74, 833

Heckman, T.M., Dahlem, M., Eales, S. A., Fabbiano, G., Weaver, K. 1996, ApJ 457, 616

Heckman, T.M., Dahlem, M., Lehnert, M.D., Fabbiano, G., Gilmore, D., Waller, W.H. 1995, ApJ 448, 98

Lehnert, M.D., Heckman, T.M. 1996a, ApJ 462, 651

Lehnert, M.D., Heckman, T.M. 1996b, ApJ 472, 546

Lehnert, M.D., Heckman, T.M., Weaver, K.A. 1999, ApJ submitted

Meurer, G.R., Heckman, T.M., Lehnert, M.D., Leitherer, C., Lowenthal, J. 1997, AJ 114, 54

Moran, E.C., Lehnert, M.D. 1997, ApJ 478, 172

Moran, E.C., Lehnert, M.D., Helfand, D. 1999, ApJ in press

Norman, C.A., Bowen, D.V., Heckman, T.M., Blades, C., Danly, L. 1996, ApJ 472, 73

Persic, M., Mariani, S., Cappi, M. et al. 1998, A\&A 339, 33

Ptak, A., Serlemitsos, P., Yaqoob, T., Mushotzky, R., Tsuru, T. 1997, AJ 113, 1286

Strickland, D.K., Ponman, T.J., Stevens, I.R. 1997, A\&A 320, 378

Suchkov, A. A., Balsara, D., Heckman, T. M., Leitherer, C. 1994, ApJ 430, 511

Suchkov, A.A., Berman, V.G., Heckman, T.M., Balsara, D. 1996, ApJ 463, 528

Tenorio-Tagle, G., Muñoz-Tuñón, C. 1997, ApJ 478, 134

Tenorio-Tagle, G., Muñoz-Tuñón, C. 1998, MNRAS 293, 299

Watson, M.G., Stanger, V., Griffiths, R.E. 1984, ApJ 286, 144

Weedman, D.W., Wolovitz, J.B., Bershady, M.A., Schneider, D.P. 1998, AJ 116, 1643

Yun, M.S., Ho, P.T.P., Lo, K.Y. 1993, ApJ 411, L17

Yun, M.S., Ho, P.T.P., Lo, K.Y. 1994, Nature 372, 530 